To cite this article: P. Mendes, K. Lorenz, E. Alves, S. Schwaiger, F. Scholz, S. Magalhães, Materials Science and Semiconductor Processing, 2019, 98, 95-99

DOI: 10.1016/i.mssp.2019.04.001

\title{
Measuring strain caused by ion implantation in GaN
}

\author{
P. Mendes ${ }^{1}$, K. Lorenz ${ }^{1,2}$, E. Alves ${ }^{1}$, S.Schwaiger ${ }^{3}$, F.Scholz $^{3}$, S. Magalhães*1 \\ ${ }^{1}$ Instituto de Plasmas e Fusão Nuclear, Instituto Superior Técnico, Universidade de Lisboa, Av. Rovisco Pais, 1049-001, \\ Lisboa, Portugal, \\ ${ }^{2}$ INESC-MN, Rua Alves Redol, 9 1000-029 Lisboa, Portugal \\ ${ }^{3}$ Institute of Optoelectronics, University of Ulm, Albert-Einstein-Allee 45, D-89069, Ulm, Germany
}

The reaction of $\mathrm{GaN}$ to ion implantation was studied in thin films grown on the a-plane (non-polar) and on the c-plane (polar). $300 \mathrm{keV}$ argon ions were implanted at room temperature to fluences ranging from $5 \times 10^{12}$ atoms $/ \mathrm{cm}^{2}$ to $8 \times 10^{15}$ atoms $/ \mathrm{cm}^{2}$. The structural analysis was performed using Rutherford Backscattering/Channeling and X-Ray Diffraction. The results allow to reinforce the suggestion that perpendicular strain caused by ion implantation is the driving force behind defect transformation processes inside the lattice. Furthermore, they confirm a lower relative defect level for a-GaN implanted with the highest fluence, in comparison with c-GaN, as reported previously for low temperature implantation.

Keywords: Gallium Nitride, Implantation, Ion channeling, X-ray diffraction, Strain

\section{Introduction}

Wurtzite group-III nitride semiconductors grown on nonpolar and semi-polar orientations are candidates for manufacturing high performance optoelectronic devices by enabling the development of improved device active regions [1]. The reason is that quantum wells (QWs) deposited on off-polar planes suffer less from the quantum confined Stark effect. This effect is originated by immobile interfacial charges impelled by the polarization discontinuity along the c-axis [2-6]. Nonpolar $\mathrm{GaN}$ is expected to enable higher quantum efficiencies and improved electrical characteristics for light emitting diodes (LEDs) and laser diodes. However, the ouput power of non-polar QW LEDs, especially with increasing In-cation incorporation, fabricated on heteroepitaxial substrate has been lower $[7,8]$ than the conventional c-plane LEDs due to the presence of high densities of basal stacking faults $[9,10]$. One of the hindrances of non-polar and semi-polar growth is that these extended defects scarcely vanish and rather propagate through the whole of the epilayer once they are established. Aforementioned basal stacking faults deteriorate the surface morphology by roughing it and give rise to macroscopic compositional inhomogeneities in compound films [11-14] on the grounds that the cation incorporation efficiency diverges depending on the crystallographic orientation of the growth [15]. On the one hand, crystallographic defects, affect locally the crystalline structure via complicated relaxation mechanisms by changing the interatomic distances. On the other hand, they may interact with defects created during device processing. In particular ion implantation has been applied in non-polar laser structures to form planar appertures for lateral light confinement [16]. In fact, ion implantation allows the controlled introduction of point defects within a specific sample volume for defect engineering, in this case by changing the refractive index. The effects of ion implantation into $\mathrm{GaN}$ have been widely studied in c-plane $\mathrm{GaN}$ and are characterized by strong dynamic annealing [17,18], i.e. point defects created during the implantation are very mobile and can either recombine or interact to form extended defects such as stacking faults and dislocation loops during the implantation process $[19,20]$. It has been suggested that strain is the driving force for defect transformation in $\mathrm{GaN}$ [17,18]. Strain, or more precisely the deformation perpendicular to the sample surface caused by implantation of a thin surface layer, can be directly measured via X-ray diffraction (XRD) which is very sensitive to small variations in the lattice parameter [21-24]. It was reported previously that the defect nature of extended defects caused by high fluence ion implantation is different in a-plane and c-plane $\mathrm{GaN}$ [25]. Defect transformation at high fluences can be 
influenced by distinct defects and strain states in the asgrown material or by different strain states caused by the ion implantation itself. In this work, polar and non-polar GaN samples were implanted with different fluences in order to understand how strain is propagated in both structures. Argon implantation into the GaN host lattice allows the comparative study of how strain develops for both polar and non-polar GaN focusing on the crystalline quality on which defects constitute the dominant player and chemical interactions are disregarded. Routine codes for the simulation of the XRD radial scans were used. The codes use the dynamical theory for XRD and provide the deformation perpendicular to the sample surface as function of depth [26,27].

\section{Experimental Details}

GaN thin films were grown by MOVPE - Metal Organic Vapor Phase Epitaxy. We used the standard precursors ammonia $\left(\mathrm{NH}_{3}\right)$, trimethylgallium (TMGa) and trimethylaluminum (TMAl) and Pd-diffused $\mathrm{H}_{2}$ as carrier gas. Non-polar a-plane and polar c-plane $\mathrm{GaN}$ samples have been deposited on r-oriented [28] and coriented [29] sapphire $\left(\mathrm{Al}_{2} \mathrm{O}_{3}\right)$ substrates, respectively, using an AlN nucleation layer. SiN nanomask layers were also incorporated to reduce the dislocation density. Argon was implanted at room temperature, with energy of $300 \mathrm{keV}$ and a $7^{\circ}$ incident angle between the beam and the sample normal to minimize channeling effects. According to SRIM [30] Monte Carlo simulations the maximum range of argon ions extends to $380 \mathrm{~nm}$. The projected range for Ar is at a depth of $190 \mathrm{~nm}$, while the maximum of the vacancy profile is predicted to occur at around $130 \mathrm{~nm}$ depth. Therefore, the crystal thickness is much larger than the implanted area and will impose a biaxial strain rather than relaxation through creation of defects. The implantation of Argon in GaN was performed at LATR, Campus Tecnológico e Nuclear of IST, Bobadela, Portugal. a-plane and c-plane samples were implanted simultaneously to fluences of $5 \times 10^{12}$ $\mathrm{at} / \mathrm{cm}^{2}, 5 \times 10^{13} \mathrm{at} / \mathrm{cm}^{2}, 2 \times 10^{14} \mathrm{at} / \mathrm{cm}^{2}, 5 \times 10^{14} \mathrm{at} / \mathrm{cm}^{2}$, $1 \times 10^{15} \mathrm{at} / \mathrm{cm}^{2}, 2 \times 10^{15} \mathrm{at} / \mathrm{cm}^{2}$ and $8 \times 10^{15} \mathrm{at} / \mathrm{cm}^{2}$ at room temperature. The fluences were chosen to cover several defect accumulation regimes described earlier [25] while avoiding very high fluences at which the diffraction intensity and thus the sensitivity of XRD to implantation damage is strongly reduced [31]. Rutherford backscattering spectrometry and ion channeling were measured in a Van de Graaff accelerator with $2.0 \mathrm{MeV} \alpha$ particles. X-ray diffraction was measured in a Bruker D8 AXS diffractometer equipped with a Göebel mirror and a (220) Ge monochromator to decrease the beam divergence and select $\mathrm{K} \alpha_{1}$ radiation. In front of the scintillation detector a $0.1 \mathrm{~mm}$ wide slit was placed.

\section{Results and discussion}

The as-grown samples for both planar orientations exhibit very good crystal qualities, revealing minimum yield values from RBS/C measurements around $2 \%$ for a-GaN and $2.6 \%$ for $\mathrm{c}-\mathrm{GaN}$ - which corresponds to typical values measured for state-of-the-art epitaxial GaN films. The values are determined for a region close to the surface. The relative damage level (RDL) profiles shown in Figure 1, which plot the relative concentration of displaced lattice atoms versus depth, were determined by using a code that implements a two-beam model [19] in order to account for the dechanneling background. Part of the ion beam is channeled with strongly reduced backscattering yield while the other component interacts with the crystal in a similar way as for amorphous material due to scattering by displaced atoms, namely point and extended defects, or thermally displaced atoms.

Although for low fluences ion channeling at room temperature shows low sensitivity to damage production, the first row in Fig. 1 (regime I) shows a continuous increase of the defect level with fluence. The shape of the defect profile agrees well with the SRIM simulation of the vacancy profile for both planar orientations. In regime II, it is possible to identify a saturation of the defect level for both planar orientations. The RDL stays below 0.03 for the c-plane orientation, while the saturation RDL is slightly higher for a-plane oriented samples. This very small difference can, however, be an artefact of the dechanneling correction using the twobeam model which does not take into account the different nature of extended defects in both materials [32]. In the third regime (row III in Fig. 1), the shape of the defect profile clearly deviates from the SRIM simulations for both materials, an occurrence which has been reported previously for c-GaN $[18,31]$ and a-GaN for low temperature implantation [25]. The damage close to the surface is much lower than expected - suggesting a migration and recombination of defects. For a-GaN, additionally, the depth of maximum damage changes considerably and it is found at the depth of maximum Ar-concentration.

The different damage accumulation regimes are similar to those reported previously where the first linear increase was attributed to point defect formation due to well separated ion impacts. In regime II, defect cascades start to overlap leading to increased recombination of point defects and a saturation of the defect level. For higher fluences the formation of extended defects such as stacking faults and dislocation loops sets in [25]. Furthermore, for the highest fluence the RDL is considerably higher in $\mathrm{c}-\mathrm{GaN}$ than in a-GaN, a fact that has been previously seen for Ar implantation at low temperature [25] as well as for rare earth implantation at room temperature $[33,34]$. 


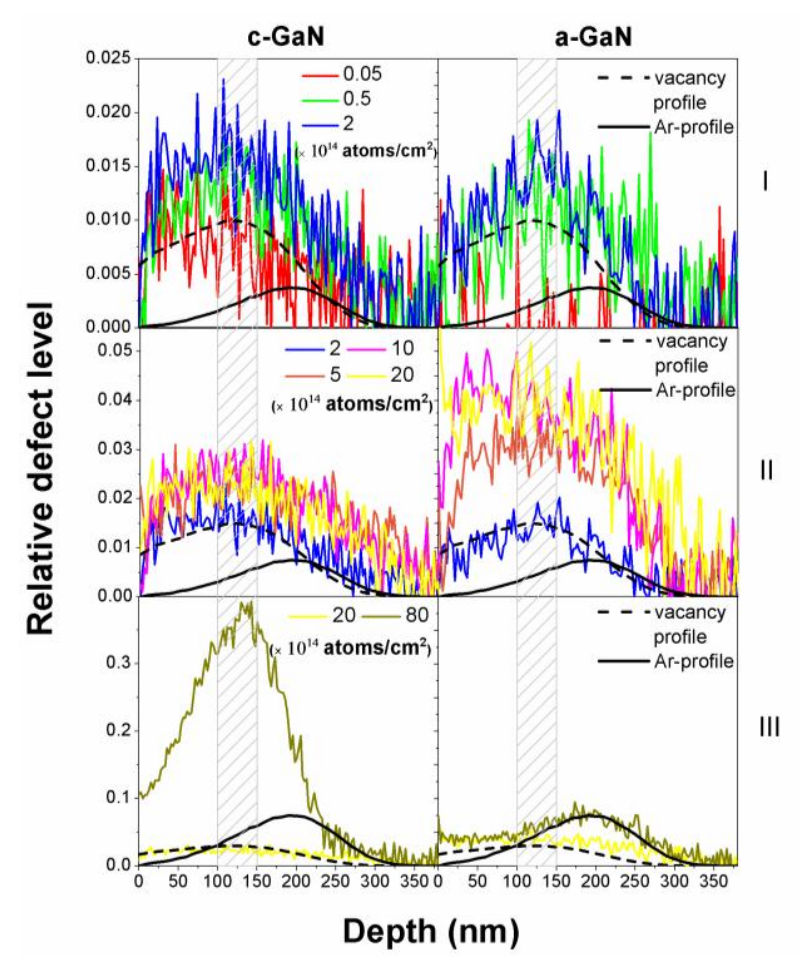

Figure 1 - Relative defect level profiles derived from the RBS/C spectra using a two-beam model for both planar orientations. Roman numbers on the right side of each row indicate the three different damage build-up regimes discussed in the text. The distributions of argon (solid lines) and vacancies (dashed lines) simulated using the SRIM code [30] are included for comparison, with arbitrary units. Fluences are given in units of $10^{14} \mathrm{at} / \mathrm{cm}^{2}$. The area between $100 \mathrm{~nm}$ and $150 \mathrm{~nm}$ is highlighted as it corresponds to the depth window used to plot the damage buildup in Fig. 4.

To study the induced strain in both planar orientations, XRD analysis was performed for all implanted fluences, measuring $2 \theta-\omega$ scans of symmetric reflections; (0002) for $\mathrm{c}-\mathrm{GaN}$ and for (112̄0) a-GaN for a-GaN, respectively. Expansion of the lattice due to ion implantation occurs only in the direction perpendicular to the surface in c-GaN and a-GaN [25].

With respect to the virgin samples, both FWHM of the peak are below $0.02^{\circ}$ for mentioned reflections confirming the very good crystalline quality derived via ion channelling measurements.

Figure 2 presents the $2 \theta-\omega$ scans for both crystal orientations. After implantation, satellite peaks are formed for lower $2 \theta$ angles (higher c- and a-lattice parameters for $\mathrm{c}-\mathrm{GaN}$ and $\mathrm{a}-\mathrm{GaN}$, respectively) with respect to the main Bragg peak due to diffraction from the implanted volume. For both planar orientations, up to a fluence of $2 \times 10^{15} \mathrm{at} / \mathrm{cm}^{2}$, the satellite peaks are welldefined and suggest that homogeneously strained layers are formed. By increasing the fluence, it is possible to identify an increased perpendicular lattice strain, given by a shift on the satellite peak to lower $2 \theta$ angles. For the highest fluence $\left(8 \times 10^{15} \mathrm{at} / \mathrm{cm}^{2}\right)$, the position does not change compared to the previous fluence (at around $\varepsilon \perp=$ $0.65 \%$ and $\varepsilon^{\perp}=0.9 \%$ for $\mathrm{c}-\mathrm{GaN}$ and $\mathrm{a}-\mathrm{GaN}$, respectively) and the satellite peak broadens strongly. The sample is already strongly damaged for the highest fluence, thus, diffraction intensity is very low.
The same pattern was already observed for c-GaN implanted with $\mathrm{Ar}$ at $200 \mathrm{keV}$ where strain saturation occurs for higher fluences [31].

The diffractograms were then simulated with MROX and RaDMaX software [26,27] to accomplish a better understanding of the strain. The results of both codes are equivalent. The resulting fits are shown in Fig. 2. Figure 3 shows the strain profiles where the range of $380 \mathrm{~nm}$ for the depth axis was limited to the maximum argon range obtained from SRIM simulations.
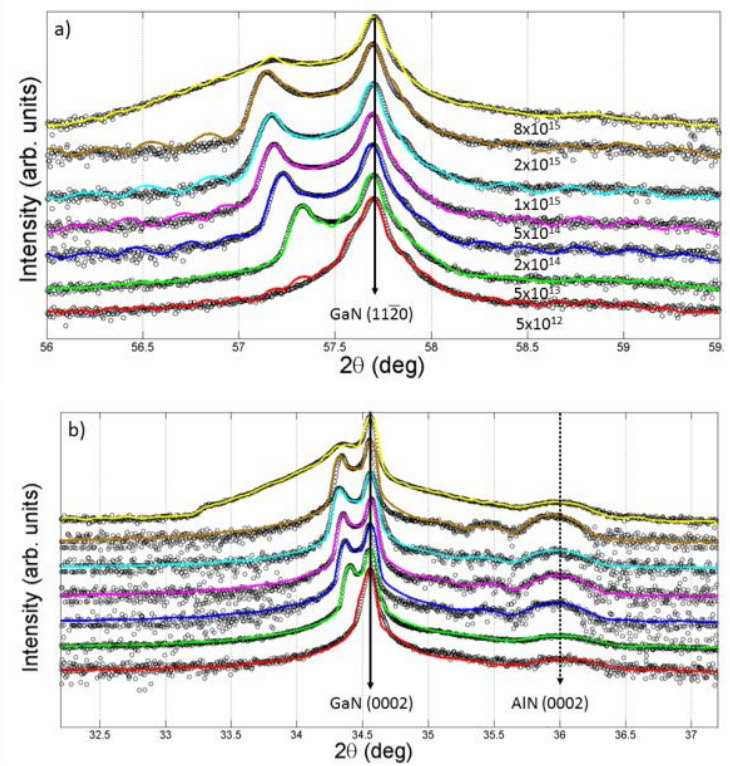

Figure 2 - XRD diffractograms (symbols) and fits (lines) using the MROX code [26] around the (0002) reflection for c-GaN (a) and $(11 \overline{2} 0)$ reflection for a-GaN (b), implanted to different fluences.

The SRIM vacancy-profile is included for comparison with arbitrary units. A good fit is achieved when using profiles similar to SRIM vacancy profile for low fluences (up until $1 \times 10^{15} \mathrm{at} / \mathrm{cm}^{2}$ ), while the transition of the strain profiles from $1 \times 10^{15}$ to $2 \times 10^{15}$ at $/ \mathrm{cm}^{2}$ may indicate a development of the strain around $270 \mathrm{~nm}$ for both planar orientations. It is clear for both planar orientations that the strain increases with the fluence. For fluences below $8 \times 10^{15} \mathrm{at} / \mathrm{cm}^{2}$, there is a wide depth region with almost constant strain, which agrees with the fact that the diffractograms show a well-defined second, lower angle peak. Furthermore, for this fluence range, the maximum strain value reached for both planar orientations agrees well with the value calculated directly from the $2 \theta$ value of the satellite peak in the diffractogram. This is illustrated for the samples implanted to $2 \times 10^{15} \mathrm{at} / \mathrm{cm}^{2}$ by the horizontal, dashed line where maximum strain values measured are $\sim 0.7 \%$ for $\mathrm{c}-\mathrm{GaN}$ and $\sim 0.95 \%$ for a-GaN. As it is possible to observe, the maximum strain is higher for a-GaN than for c-GaN for every fluence up to $2 \times 10^{15} \mathrm{at} / \mathrm{cm}^{2}$. It has, however, been previously reported that direct comparison between induced strain caused by implantation defects for different planar orientations may not be trivial. Debelle et al. [23,24] pointed out that biaxial strain due to the substrate effect has to be considered when studying induced strain following ion implantation in single crystals. In a-GaN this would lead 
to an anisotropic biaxial strain component since the inplane lattice parameters are not equivalent in contrast to c-plane material.

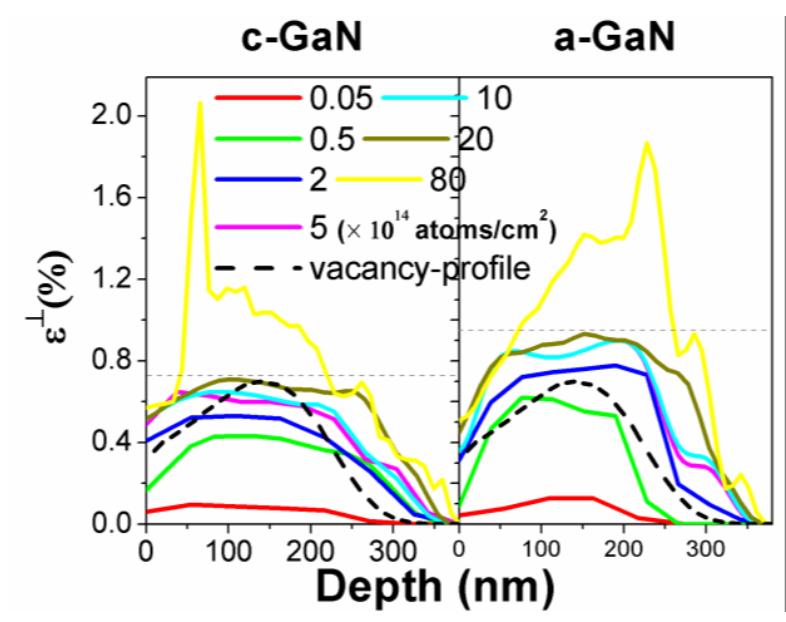

Figure 3 - Strain profiles for a) c-GaN and b) a-GaN as a function of depth. The range of $380 \mathrm{~nm}$ for the depth axis corresponds to the maximum argon atom range obtained from SRIM simulations. The SRIM vacancy-profile is included for comparison with arbitrary units.

Figure 4 gives an overview of the main parameters extracted from both techniques, RBS/C and XRD, which allows a qualitative comparison. It shows the RDL and perpendicular strain as a function of the fluence for both materials orientations.

The RBS results (RDL) are similar to previous measurments on a- and c-plane GaN for implantation at $15 \mathrm{~K}$ [25] with the difference that the transitions between different defect regimes occur at slightly higher fluences at room temperature. This is expected due to higher defect recombination and the results are in accordance with those presented by Wendler et al. [18] comparing Ar implanted c-GaN at $15 \mathrm{~K}$ and room temperature Like the relative defect level, also the strain is observed to increase with the ion fluence.

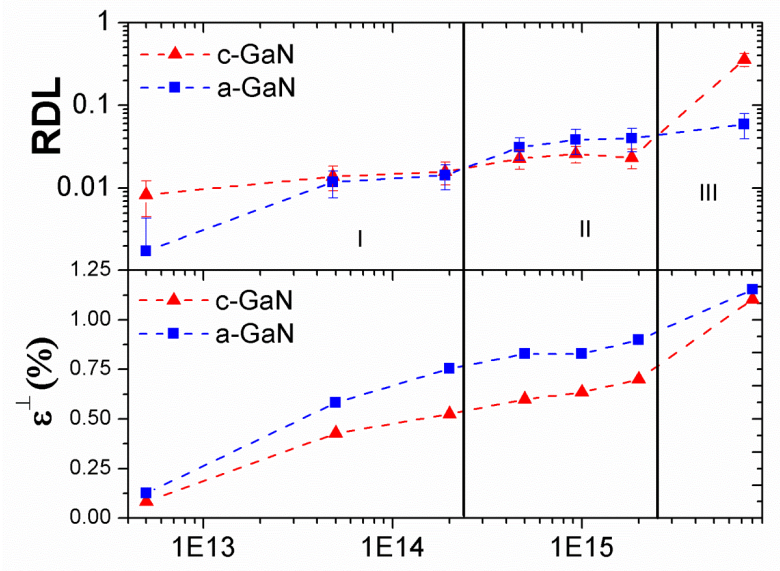

\section{Fluence}

Figure 4 - Average values of the relative defect level and strain values as a function of the fluence in the depth region from 100 to $150 \mathrm{~nm}$.
By relating the three regimes previously discussed for the RBS/C results to their strain profiles, it is possible to observe a similar development for both planar orientations: a first regime where the increase of the strain is relatively steep as the fluence rises, followed by a second regime where the increase is smaller (in agreement with the saturation of the RDL). For the third regime, for a fluence of $8 \times 10^{15} \mathrm{at} / \mathrm{cm}^{2}$, there is a significantly stronger increase of RDL value observed for $\mathrm{c}-\mathrm{GaN}$ than for a-GaN. As previously reported, the samples show a distinct defect morphology for this fluence with predominantly stacking faults in c-GaN and dislocation loops in a-GaN [25]. The strain value for this fluence, is nearly the same for both planar orientations (in contrast to the lower fluences where starin is higher in a-GaN than in c-GaN) suggesting more efficient strain relaxation in a-GaN possibly resulting in the distinct defect types. Nevertheless, our XRD data show similar behavior for the different planar orientations and thus it is not possible to determine conclusively the effect of strain on the distinct defects formed in a-GaN and cGaN.

\section{Conclusions}

Non-polar and polar oriented GaN samples were implanted with a selected set of Ar fluences from $5 \mathrm{x}$ $10^{12}$ to $8 \times 10^{15} \mathrm{at} / \mathrm{cm}^{2}$. The crystalline quality of the virgin samples is found to be state-of-the-art. Increase of fluence produces continuous increase of the interatomic distance resulting in higher deformation perpendicular to the sample surface derived from X-ray diffraction. The strain profiles were compared to damage profiles measured by ion channeling. For low fluences, both quantities follow the vacancy profile simulated with the SRIM Monte Carlo code. Both techniques suggest a 3 stage damage accumulation process within the studied fluence range. First, an increase of the deformation (and relative damage level) with fluence is observed attributed to the creation of point defects. Then, due to the high dynamic annealing typical for group-III nitride semiconductors, a saturation of defect concentration occurs. Finally, clusters of defects develop and annealing is much more difficult.

\section{Acknowledgments}

Financing by FCT Portugal and FEDER is acknowledged (PTDC/CTM-CTM/28011/2017, LISBOA-01-0145-FEDER-028011). S. Magalhães also acknowledges FCT for his post-doc grant (ref. SFRH/BPD/FIS/98738/2013).

\section{References}

[1] J. S. Speck, S. F. Chichibu, MRS Bull. 34 (2009) 304

[2] T. Takeuchi, D. Sota, M. Katsuragawa, M. Komori, H. Takeuchi, Amano, I. Akasaki, Jp. J. Appl. Phys. 36 (1997) L382

[3] S. Chichibu, T. Azuhata, T. Sota, S. Nakamura, Appl. Phys. Lett. 69 (1996) 4188

[4] T. Takeuchi, H. Amano, I. Akasaki, Jp. J. Appl. Phys. 39 (2000) 413 
[5] A. E. Romanov,T. J. Baker, S. Nakamura, J. S. Speck , J. Appl. Phys. 100 (2000) 6023522

[6] S. F. Chichibu, A. Uedono, T. Onuma, B. A. Haskell, A. Chakraborty, T. Koyama, P. T. Fini, S. Keller, S. P. DenBaars, J. S. Speck, U. K. Mishra, S. Nakamura, S. Yamaguchi, S. Kamiyama, H. Amano, I. Akasaki, J. Han, T. Sota, Nature Mater. 5 (2006) 810

[7] A. Chakraborty, B. A. Haskell, S. Keller, J. S. Speck, S. P. DenBaars, S. Nakamura S, U. K. Mishra, Appl. Phys. Lett. 85 (2004) 5143

[8] A. Chakraborty, B. A. Haskell, S. Keller, J. S. Speck, S. P. DenBaars, S. Nakamura, U. K. Mishra, Jp. J. Appl. Phys. 44 (2005) L173

[9] B. A. Haskell, F. Wu, M. D. Craven, S. Matsuda, P. T. Fini, T. Fujii, K. Fujito, S. P. DenBaars, J. S. Speck, S. Nakamura, Appl. Phys. Lett. 83 (2003) 644

[10] B. A. Haskell, T. J. Baker, M. B. McLaurin, F. Wu, P. T. Fini, S. P. DenBaars, J. S. Speck J S, S. Nakamura, Appl. Phys. Lett. 86 (2005) 111917

[11] A. Chakraborty, S. Keller, C. Meier, B. A. Haskell, A. Keller, P. Waltereit, S. P. DenBaars, S. Nakamura, J. S. Speck, U. K. Mishra, Appl. Phys. Lett. 86 (2005) 031901

[12] T. Onuma, A. Chakraborty, B. A. Haskell, S. Keller, S. P. DenBaars, J. S. Speck, S. Nakamura, U. K. Mishra, T. Sota, S. F. Chichibu, Appl. Phys. Lett. 86 (2005) 151918

[13] T. Koyama, T. Onuma, H. Masui, A. Chakraborty, B. A. Haskell, S. Keller, U. K. Mishra, J. S. Speck, S. Nakamura, S. P. DenBaars, T. Sota, S. F. Chichibu, Appl. Phys. Lett. 89 (2006) 091906

[14] T. Koyama, T. Onuma, H. Masui, A. Chakraborty, B. A. Haskell, S. Keller, U. K. Mishra, J. S. Speck, S. Nakamura, S. P. DenBaars, T. Sota, S. F. Chichibu, J. Vac. Sci. Technol. B 25 (2007) 1524

[15] R. Bhat, G. M Guryanov, J. Cryst. Growth 433 (2016) 7 12

[16] J. T. Leonard, D. A. Cohen, B. P. Yonkee, R. M. Farrell, T. Margalith, S. Lee, S. P. DenBaars, J. S. Speck, S. Nakamura. Appl. Phys. Lett. 107 (2015) 011102

[17] A. Turos, Radiat. Eff. Defects Solids 1686 (2013) 431441

[18] E. Wendler, W. Wesch, A. Yu. Azarov, N. Catarino, A. Redondo-Cubero, E. Alves, K. Lorenz, Nucl. Instr. Meth. Phys. Res. Sect. B 307 (2013) 394-398

[19] E. Wendler, W. Wesch, G. Götz, J. Appl. Phys. 70 (1991) 144-149

[20] S. O. Kucheyev, J. S. Williams, S. J. Pearton, Mater. Sci. Eng. R Reports 33 2-3 (2001) 51-107

[21] F. Gloux, T. Wojtowicz, P. Ruterana, K. Lorenz, E. Alves, J. Appl. Phys 100 (2006) 7

[22] B. Lacroix, S. Leclerc, A. Declémy, K. Lorenz, E. Alves, and P. Ruterana, J. Appl. Phys.112 (2011) 7

[23] A. Debelle, A. Declémy, Nucl. Instr. Meth. Phys. Res. Sect. B 2689 (2010) 1460-1465

[24] A. Debelle, A. Boulle, F. Rakotovao, J. Moeyaert, C. Bachelet, F. Garrido, L. Thomé, J. Appl- Phys. D: Appl. Phys. 46 (2013) 045309

[25] K. Lorenz, E. Wendler, A. Redondo-Cubero, N. Catarino. M.-P.Chauvat, S.Schwaiger, F.Scholz, P. Ruterana, Acta Mater. 123 (2017) 177-187

[26] S. Magalhães, M. Fialho, M. Peres, K. Lorenz, E. Alves, J. Phys. D. Appl. Phys 49 (2016) 135308

[27] M. Souilah, A. Boulle, and A. Debelle, J. Appl. Crystallogr. 49 (2016) 311-316

[28] J. Hertkorn, P. Brückner, S. B. Thapa, T. Wunderer, F. Scholz, M. Feneberg, K. Thonke, R. Sauer, M. Beer, J. Zweck, J. Cryst. Growth 308 (2007) 30-36

[29] S. Schwaiger, F. Lipski, T. Wunderer, F. Scholz, Phys. Status Solidi C 7 (2010) 2069-2072
[30] J. F. Ziegler, M. D. Ziegler, and J. P. Biersack, Nucl. Instr. Meth. B 268 (2010) 1818-1823

[31] D. Nd. Faye, E. Wendler, M. Felizardo, S. Magalhães, E. Alves, F. Brunner, M. Weyers, and K. Lorenz, J. Phys. Chem. C, vol. 120 (2016) 7277-7283

[32] A. Turos, P. Jozwik, L. Nowicki, N. Sathish, Nucl. Instr. Meth. B 332 (2014) 50

[33] A. Macková, P. Malinsky, A. Jágerová, Z. Sofer, D. Sedmidubský, K. Klímová, R. Böttger, S. Akmadaliev, Surf Interface Anal. (2018) 1-7

[34] N. Catarino, E. Nogales, N. Franco, V. Darakchieva, S. M. C. Miranda, B. Méndez, E. Alves, J. G. Marques, K. Lorenz, Europhysics Lett. 97 (2012) 68004 